\title{
Snail-induced epithelial-mesenchymal transition promotes cancer stem cell-like phenotype in head and neck cancer cells
}

\author{
TAKASHI MASUI ${ }^{*}$, ICHIRO OTA $^{1 *}$, JONG-IN YOOK $^{2}$, SHINJI MIKAMI $^{1}$, \\ KATSUNARI YANE $^{3}$, TOSHIAKI YAMANAKA ${ }^{1}$ and HIROSHI HOSOI ${ }^{1}$ \\ ${ }^{1}$ Department of Otolaryngology-Head and Neck Surgery, Nara Medical University, Kashihara, Nara 634-8522, Japan; \\ ${ }^{2}$ Department of Oral Pathology, Oral Cancer Research Institute, College of Dentistry, Yonsei University, \\ Seoul 120-752, Republic of Korea; ${ }^{3}$ Department of Otolaryngology, Kinki University \\ School of Medicine, Nara Hospital, Ikoma, Nara 630-0293, Japan
}

Received October 1, 2013; Accepted November 20, 2013

DOI: $10.3892 /$ ijo.2013.2225

\begin{abstract}
Head and neck squamous cell carcinoma (HNSCC) is known to have a poor prognosis. The resistance to treatment and distant metastasis are important clinical problems in HNSCC. The epithelial-mesenchymal transition (EMT) is a key process in successful execution of many steps such as the invasion and metastasis for cancer cells. Snail is one of the master regulators that promote EMT in many types of malignancies including HNSCC. Recently, it has been shown that Snail-induced EMT could induce a cancer stem cell (CSC)-like phenotype in a number of tumor types. In this study, we investigated the role of Snail in inducing EMT properties and CSC-like phenotype in HNSCC. We established HNSCC cell lines transfected with Snail. E-cadherin was analyzed using western blot analysis and immunofluorescence staining. Cell migration and invasion were assessed using wound-healing assay and modified Boyden chamber assay, respectively. CSC markers of HNSCC, CD44 and aldehyde dehydrogenase 1 (ALDH1), were also evaluated with western blot analysis, and chemosensitivity was assessed with WST-8 assay. Introduction of Snail induced EMT properties in HNSCC cells and enhanced cell migration and invasion. Moreover, Snail-induced EMT gained CSC-like phenotype and was associated with increased chemoresistance. These
\end{abstract}

Correspondence to: Dr Ichiro Ota, Department of OtolaryngologyHead and Neck Surgery, Nara Medical University, 840 Shijo-cho, Kashihara, Nara 634-8522, Japan

E-mail: iota@naramed-u.ac.jp

${ }^{*}$ Contributed equally

Abbreviations: HNSCC, head and neck squamous cell carcinoma; EMT, epithelial-mesenchymal transition; ALDH1, aldehyde dehydrogenase 1; DAPI, 4',6-diamidino-2-phenylindole; CSC, cancer stem cell

Key words: epithelial-mesenchymal transition, Snail, cancer stem cell, head and neck cancer results suggest that Snail could be one of the attractive targets for the development of therapeutic strategies in HNSCC.

\section{Introduction}

Head and neck squamous cell carcinoma (HNSCC) is the sixth most common type of cancer in the world and also known for its rapid clinical progression and poor prognosis $(1,2)$. The mortality is mainly caused by locoregional recurrence and cervical lymph node metastasis and occasionally by distant metastasis (3). Notably, regional and distant metastases in HNSCC correspond to an extremely poor prognosis with limited treatment options. The treatment resistance and tumor recurrence are important clinical problems in the management of HNSCC. To improve the therapeutic outcome of HNSCC, more effective treatment strategy is urgently needed.

Epithelial-mesenchymal transition (EMT) is a critical process in tumor progression that causes epithelial cells to acquire a migratory mesenchymal phenotype $(4,5)$. EMT is thought to be a crucial step in the induction of cell invasion and tumor metastasis (4). Furthermore, it has also been shown that cells with an EMT phenotype are more resistant to chemoradiotherapy in HNSCC (6).

Snail, a zinc-finger transcription factor, plays an important role in EMT by directly repressing epithelial marker such as E-cadherin and by upregulating mesenchymal markers (7-12). Several studies have shown that Snail-related transcription factors play a transcriptional and regulatory role in invasion, metastasis, and poor outcome for different type of malignancies, including HNSCC $(13,14)$.

It has been suggested in recent reports that Snail-induced EMT causes the cancer stem cell (CSC)-like properties in different type of malignant tumors and that both EMT and CSC-like phenotype are associated with treatment resistance $(11,15,16)$. Prince et al showed that the purified CD $44^{+}$population of HNSCC cells possesses the self renewing properties of CSCs (17). Aldehyde dehydrogenase 1 (ALDH1) has also been shown to be a putative marker of CSC in HNSCC (18-20). Furthermore, Chen et al showed that $\mathrm{CD} 44^{+} / \mathrm{ALDH}^{+}{ }^{-}$cells 
resist radiotherapy and may serve as a reservoir for developing tumors and metastasis (21). These findings suggested that Snail expression may regulate the CSC-like properties in HNSCC via EMT. On the contrary, there is also a report that Snail expression did not correlate with prognosis (22).

The key role of Snail in HNSCC has not been fully elucidated. In this study, we demonstrate that introduction of Snail in HNSCC cells confers EMT properties such as increased cell motility and invasiveness in vitro. In addition, we report that Snail-induced EMT gains HNSCC cell CSC-like phenotype and are associated with chemoresistance.

\section{Materials and methods}

Cell lines and culture. Human HNSCC cells, SAS and HSC-4, were employed in this study. SAS cells and HSC-4 cells, obtained from the Japanese Cancer Research Resource Bank (Tokyo, Japan), were cultured in DMEM medium (Invitrogen, Carlsbad, CA, USA) supplemented with $10 \%$ heat-inactivated fetal bovine serum (FBS, Invitrogen), $100 \mathrm{U} / \mathrm{ml}$ penicillin, and $100 \mathrm{mg} / \mathrm{ml}$ streptomycin (Gibco, Grand Island, NY, USA) at $37^{\circ} \mathrm{C}$ in $5 \% \mathrm{CO}_{2}$.

Transfection with Snail in SAS and HSC-4 cells. cDNA fragment encoding human Snail (NM_005985.2) was inserted into pCR 3.1 mammalian expression vector (Invitrogen). SAS and HSC- 4 cells $\left(1.5 \times 10^{5}\right.$ cells $)$ were plated into 6 -well culture plates and allowed to adhere for $12 \mathrm{~h}$. Then, SAS and HSC- 4 cells were transfected with $2 \mu \mathrm{g}$ of either pCR 3.1-Snail or pCR 3.1-vector (without insert DNA) with Lipofectamine 2000 reagent (Invitrogen) according to the manufacturer's instructions. We established SAS-Snail and HSC-4-Snail as transiently Snail-expressing cell lines, and their respective control cell lines. All assays were performed $24 \mathrm{~h}$ after transfection.

Immunoblot analysis and antibodies. Snail, E-cadherin, CD44 and ALDH1 signaling on SAS and HSC-4 cells after the transfection with or without Snail were evaluated with western blot analysis. Cells were collected and frozen in $100 \mu 1$ RIPA buffer, and stored at $-30^{\circ} \mathrm{C}$. Briefly, total protein extracts were prepared according to the freeze-thawing lysis method and protein concentrations were measured with Bovine Serum Albumin (BSA) Protein Assay. Sample of extract containing $20 \mu \mathrm{g}$ of protein were then separated by sodium dodecylsulfate-polyacrylamid gel electrophoresis (SDS-PAGE) and transferred to polyvinylidene difluoride membranes, after washing with phosphate-buffered saline with Tween-20 (PBST), the membranes were incubated first with rabbit anti-Snail, rabbit anti-E-cadherin (Cell Signaling Technology, Danvers, MA, USA; diluted 1:1,000), rabbit anti-CD44 and goat anti-ALDH1 (Abcom, Cambridge, MA, USA; diluted 1:2,000 and 1:500, respectively) at $4^{\circ} \mathrm{C}$ overnight and then with peroxidase-conjugated secondary anti-rabbit or goat immunoglobulin G (IgG) (Cell Signaling Technology; diluted 1:1,000) for $1 \mathrm{~h}$. After rinsing in PBST (4 times, 5 min each), immunodetection was accomplished using an ECL western blot analysis detection reagent and analysis system. The membranes were subsequently exposed to X-ray film as described previously (23).
Immunofluorescence staining. Cells were cultured in Labtech chamber slide system (Thermo Scientific, Waltham, MA, USA), and then fixed with $4 \%$ paraformaldehyde for $20 \mathrm{~min}$ at room temperature. After rinsing with phosphate-buffered saline (PBS), the cells were permeabilized with $0.1 \%$ Triton $\mathrm{X}-100$ in PBS for $30 \mathrm{~min}$. Then, they were blocked with $1 \%$ BSA and $0.1 \%$ Tween-20 in PBS for $1 \mathrm{~h}$ at room temperature and incubated overnight at $4{ }^{\circ} \mathrm{C}$ with rabbit anti-E-cadherin antibody (diluted 1:200). After rinsing with $0.1 \%$ Tween-20 in PBS, chamber slides were incubated with fluorescent-labeled secondary antibody (goat anti-rabbitIgG-Alexa Fluor 594; Invitrogen; diluted 1:1,000) for $1 \mathrm{~h}$ at room temperature in the dark. The slides were then mounted with Prolong gold antifade Reagent with 4',6-diamidino2-phenylindole (DAPI; Invitrogen). The fluorescent images were visualized by confocal image microscopy (Keyence, Osaka, Japan).

Wound healing migration assay. Equal number of cells was plated onto 24-well culture plates in DMEM medium and cultured for $24 \mathrm{~h}$. The cell monolayer was scraped with a 200- $\mu 1$ pipette tip, washed with PBS and changed to fresh medium. The wound closure was photographed at $24 \mathrm{~h}$ after wounding with control at $0 \mathrm{~h}$ under phase contrast microscope. The wound width was measured in three points per image. This experiment was repeated at least three times on each cell line. The cell migration potency was determined by calculating a difference between wound width at 0 and $24 \mathrm{~h}$.

Invasion assay. Invasion assays were performed using 24-well Matrigel-coated Transwells (BD Bioscience, Bedford,

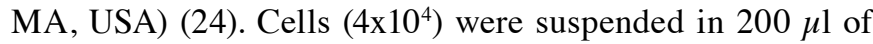
serum-free DMEM medium and placed in the top chambers, and $700 \mu \mathrm{l}$ DMEM medium containing 10\% FBS was added to the bottom chambers. After $24 \mathrm{~h}$ of incubation at $37^{\circ} \mathrm{C}$, non-invading cells were removed from the top of the Matrigel with a cotton swab, while invading cells on the bottom surface of the filter were fixed in $4 \%$ paraformaldehyde and stained with Giemsa (Sigma-Aldrich, Dorset, UK) for $30 \mathrm{~min}$. The invading cells were then visualized at x200 magnification and counted in five fields for each filter.

Chemotherapy for cultured cells. In chemotherapy, cells were treated with cisplatin (Nihonkayaku Co., Tokyo, Japan) at concentration of 1.0 or $10 \mu \mathrm{M}$. Chemosensitivity was assessed by Cell Counting Kit- 8 (WST- 8 cleavage; Dojindo, Mashikimachi, Japan) as described previously (23). The cell viability after the chemotherapy for the cells was evaluated with the WST-8 cleavage. The cells were seeded in 96-well plates at an initial density of $4 \times 10^{3}$ cells/well and incubated for $24 \mathrm{~h}$. For chemotherapy, cisplatin $(0-10 \mu \mathrm{M})$ was added to each well. Following incubation for an additional $48 \mathrm{~h}, 10 \mu \mathrm{l}$ of WST-8 solution [2-(2-methoxy-4-nitrophenyl)-3-(4-nitrop henyl)-5-(2,4-disulfophenyl)-2H-tetrazolium, monosodium salt] was added to each well, and the plate was incubated for further $2 \mathrm{~h}$. The absorbance of each well at $450 \mathrm{~nm}$ (reference wave length at $620 \mathrm{~nm}$ ) was measured by a Multiscan FC Microplate Photometer (Thermo Scientific). The measurement was repeated at least three times for each cell line. 
SAS

A

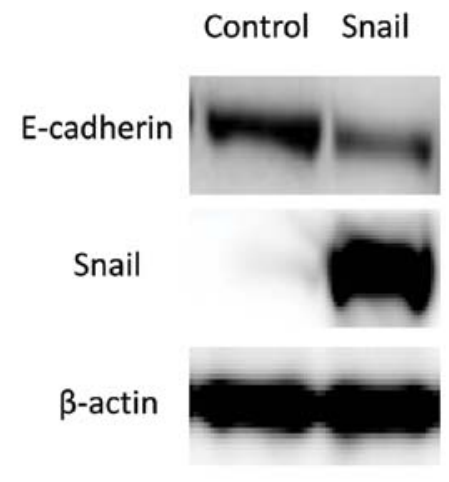

\section{C}

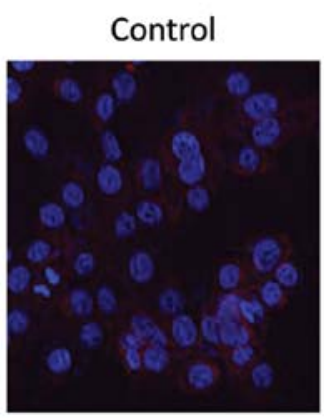

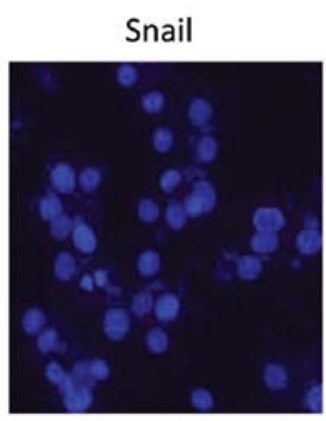

HSC-4

B

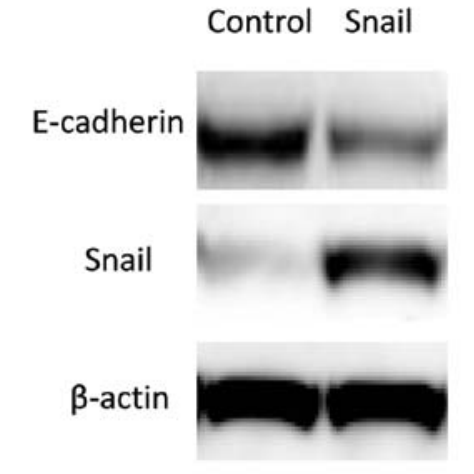

D

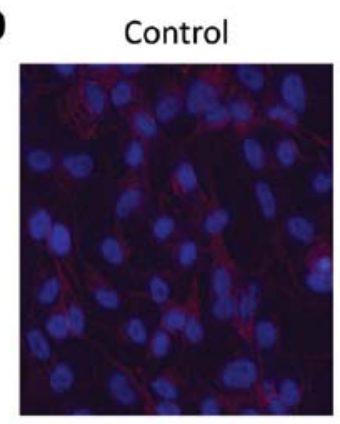

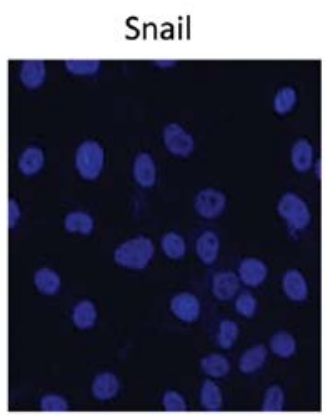

Figure 1. Snail regulates E-cadherin expression. SAS and HSC-4 cells were transfected with control or Snail expression vector. (A and B) E-cadherin and Snail protein levels were determined by immunoblot analysis using $\beta$-actin as the loading control. (C and D) Cells were stained with E-cadherin antibody [red, E-cadherin; blue, 4',6-diamidino-2-phenylindole (DAPI)-stained nuclei].

Statistical analysis. Data were presented as mean \pm standard error (SE). Experimental differences between groups were assessed with the t-test. The differences were considered to be significant at $\mathrm{P}<0.05$.

\section{Results}

Snail regulates EMT properties in HNSCC cells. SAS and HSC-4 cells were tranfected with Snail or control vector. In western blot analysis, introduction of Snail enhanced the suppression of E-cadherin protein levels in SAS and HSC-4 cells (Fig. 1A and B). In addition, cellular staining pattern of E-cadherin was examined by immunofluorescence analysis. E-cadherin staining also decreased on the cell membrane in SAS-Snail and HSC-4-Snail cells, whereas each control cell line stained positively for E-cadherin (Fig. 1C and D).

A wound healing migration assays showed that migrating cells significantly increase in SAS-Snail and HSC-4-Snail cells more than those in the control cells (Fig. 2). Furthermore, in invasion assays, both these cell lines tranfected with Snail displayed more invasive ability compared to the control cells with significant difference (Fig. 3). These results suggested that Snail was able to induce EMT in HNSCC cells.

Snail expression induces CSC-like phenotype in HNSCC cell lines. We demonstrated that EMT by Snail expression induced a stem cell-like phenotype in HNSCC cells. The expression of CSC surface markers in HNSCC cells was evaluated with western blot analysis. Both CD44 and ALDH1 protein levels increased in SAS-Snail and HSC-4-Snail cells compared with their control cells (Fig. 4). These results implied that Snailinduced EMT could elicit a CSC-like phenotypic change as CD $44^{+} / \mathrm{ALDH}^{+}$in $\mathrm{HNSCC}$ cells.

Snail expression enhances chemoresistance. The cells transfected with Snail showed significantly low chemosensitivity at 1.0 and $10 \mu \mathrm{M}$, as compared with the control cells (Fig. 5). Thus, these results implied that the acquisition of CSC-like phenotype caused by EMT results in enhancement of chemoresistance in HNSCC cells.

\section{Discussion}

In a variety of solid human tumors, the capacity to initiate and maintain cancer growth and recurrence has been found to reside in the small populations of cells within tumors, termed cancer stem cells (CSCs). CSCs have the ability to undergo self-renewal and produce differentiated progeny. These characteristics allow CSCs to maintain a pluripotent phenotype, while also producing a tumor composed of a heterogeneous cell population $(25,26)$. Several studies have shown that CSC is implicated in tumor invasion and metastasis, and that tumor recurrence after therapy is correlated with therapeutic resistance of CSCs (27-30). CSC populations in HNSCC were first identified using CD44, which has been used as a marker of CSC in different type of malignant tumors including HNSCC 
SAS

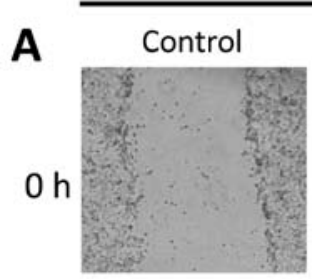

$24 \mathrm{~h}$

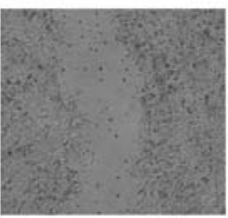

C

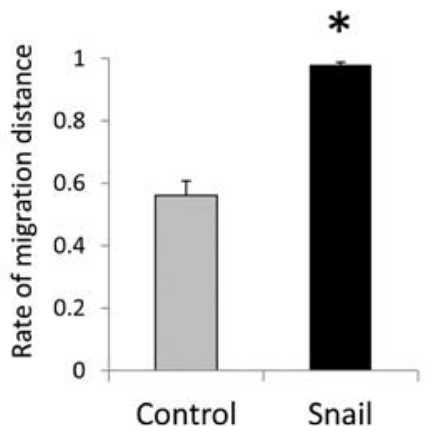

Snail
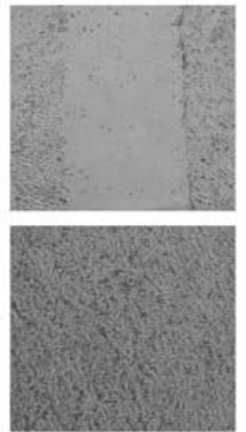

HSC-4

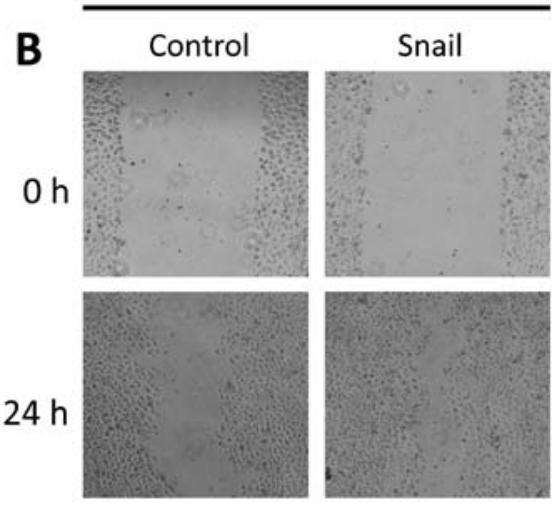

D

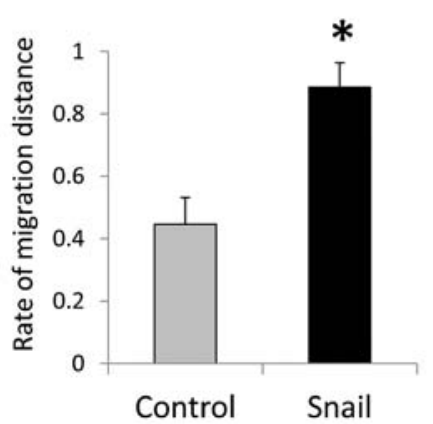

Figure 2. Snail expression increases cell migration in SAS and HSC-4 cells. (A and B) Phase contrast images were taken at 0 and $24 \mathrm{~h}$ after wounding. (C and D) The migration potency was determined by calculating a difference of wound width. Data are shown as mean $\pm \mathrm{SE}$. ${ }^{*} \mathrm{P}<0.05$.

SAS

A

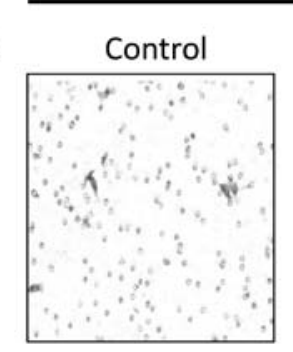

C

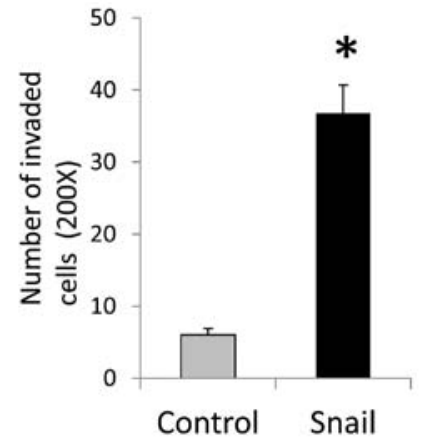

HSC-4

B

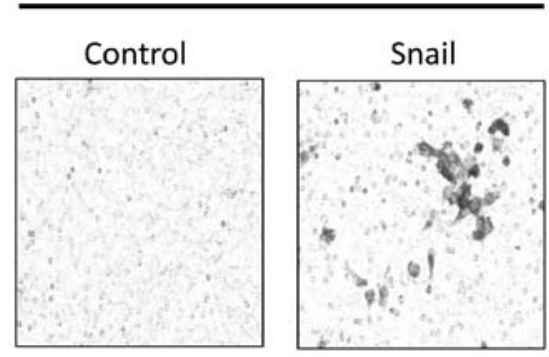

D

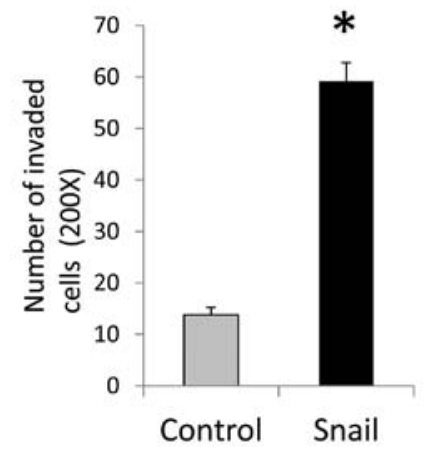

Figure 3. Snail expression increases cell invasion in SAS and HSC-4 cells. (A and B) Invading cells were visualized at x200 magnification. (C and D) Number of invading cells was counted in five fields for each filter. Results are expressed as mean $\pm \mathrm{SE}$. $\mathrm{P}<0.05$.

(17,31,32). However, HNSCC in CSCs are not precisely defined by CD44 expression alone (33). Recently, ALDH1 has been shown to be a marker of CSC. ALDH1 has also been used to identify the CSCs (34). In addition, Kirshnamurthy et al found that the combination of CD44 and ALDH1 is more selective for CSC populations than either marker used alone (35). 
SAS

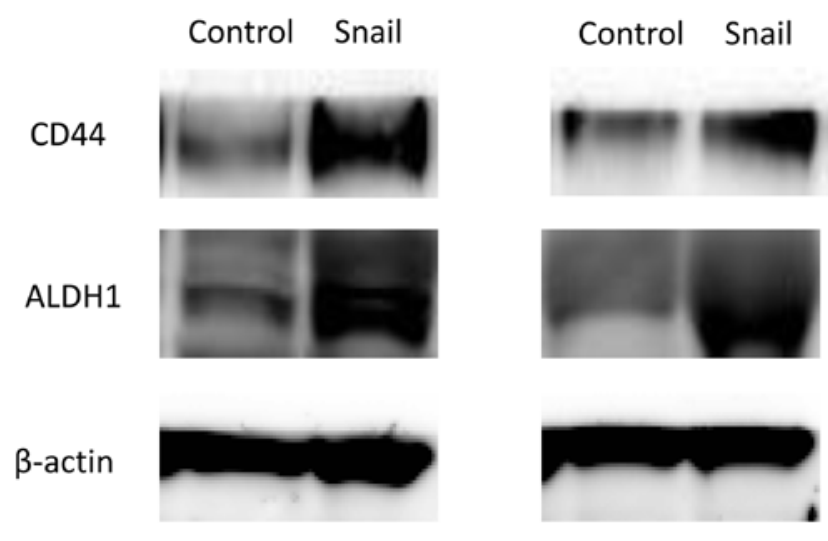

Figure 4. Snail induces expression of cancer stem cell (CSC) markers in SAS and HSC-4 cells. CD44 and aldehyde dehydrogenase 1 (ALDH1) protein levels were determined by western blot analysis with $\beta$-actin used as the internal control.

Epithelial tumor cells that undergo EMT lose cell-cell adhesion properties and acquire more mesenchymal properties, including invasiveness, motility and increase resistance to apoptosis. EMT is an important biological process that plays a critical role in tumor cell invasion, metastasis and recurrence, and is commonly observed in tumor samples from HNSCC patients $(36,37)$. Moreover, the connection between CSC and EMT has become more evident. It has been described that induction of EMT results in cells gaining CSC-like properties and treatment resistance (16,38-40). Therefore, the importance of EMT in treatment resistance has recently been targeted for investigation of CSCs in different type of cancers, including HNSCC.

Snail is one of the master regulators that promotes EMT by repressing epithelial markers and upregulating mesenchymal markers and that mediates invasiveness as well as metastasis in many different types of malignant tumors including HNSCC
(41). Furthermore, it has been reported that Snail expression may regulate the treatment resistance and CSC-like properties of HNSCC $(34,42)$. Medelsohn et al have recently reported that Snail is an independent marker of tumor metastasis in patients with HNSCC (43).

In this study, we showed that induction of Snail could suppress E-cadherin expression and increase motility and invasiveness of HNSCC cells. These results suggested that Snail could promote EMT and mediate tumor invasion. In addition, we demonstrated that induction of EMT via Snail could lead HNSCC cells to adopt CSC-like phenotype and chemoresistance for cisplatin. Previous data imply that CSCs also rely on a microenvironment, called the CSC niche, which controls their differentiation and proliferation (44-47). The CSC niche has a complex anatomical unit and is composed of diverse stromal cells, such as a vascular network, mesenchymal and immune cells, extracellular matrix (ECM), and soluble factors derived from niche cells (47-50). It has been suggested that interactions of CSC with CSC niche could induce tumor invasion and treatment resistance. The detail of interactions between CSCs and their niche are still unknown, and so the precise mechanisms should be verified in further investigation. However, understanding the interactions between the CSCs by Snail-induced EMT and their niche microenvironments, which contribute to treatment resistance, may pave the way for the development of novel strategies for treating cancer including HNSCC.

In summary, we obtained EMT properties by the overexpression of Snail in HNSCC cells. Moreover, these data suggest that Snail also acquires CSC-like phenotype via EMT and enhances treatment resistance. This Snail-induced EMT is considered to play an essential role in tumor progression and treatment resistance of HNSCC. Although the precise involvement of EMT and CSC by Snail remains to be elucidated, they could be involved in the latent effect. The critical mechanisms still need to be further investigated. However, the strategy targeting EMT-regulating Snail could be useful for cancer treatments, as the inhibition of EMT may serve
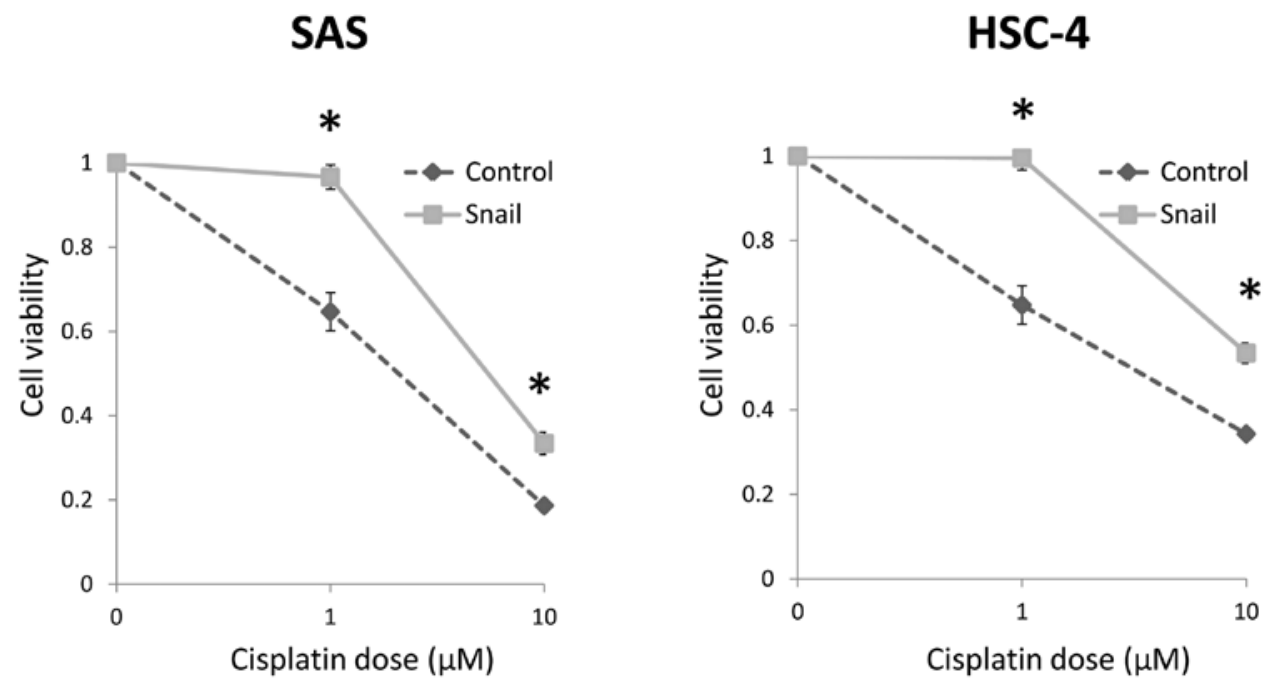

Figure 5. Snail expression enhances chemoresistance for cisplatin in SAS and HSC-4 cells. Cell viability was assessed using WST-8 assay for chemosensitivity. Cells were incubated for $24 \mathrm{~h}$ at first. For chemotherapy, cells were treated with various concentration of cisplatin. Following an additional $48 \mathrm{~h}$, cell viability was determined by WST- 8 assay. Data are shown as mean \pm SE. ${ }^{*} \mathrm{P}<0.05$. 
to block not only cancer invasion and metastasis but also the formation of CSC.

\section{Acknowledgements}

This study was supported in part by Grants-in-Aids for Scientific Research from the Ministry of Education, Culture, Sports, Science and Technology of Japan.

\section{References}

1. Ang KK, Trotti A, Brown BW, et al: Randomized trial addressing risk features and time factors of surgery plus radiotherapy in advanced head-and-neck cancer. Int J Radiat Oncol Biol Phys 51: 571-578, 2001.

2. Ozer E, Grecula JC, Agrawal A, et al: Long-term results of a multimodal intensification regimen for previously untreated advanced resectable squamous cell cancer of the oral cavity, oropharynx, or hypopharynx. Laryngoscope 116: 607-612, 2006.

3. Lo WL, Kao SY, Chi LY, et al: Outcomes of oral squamous cell carcinoma in Taiwan after surgical therapy: factors affecting survival. J Oral Maxillofac Surg 61: 751-758, 2003.

4. Thiery JP and Sleeman JP: Complex networks orchestrate epithelial-mesenchymal transitions. Nat Rev Mol Cell Biol 7: 131-142, 2006.

5. Hay ED: The mesenchymal cell, its role in the embryo, and the remarkable signaling mechanisms that create it. Dev Dyn 233 706-720, 2005

6. Hsu DS, Lan HY, Huang CH, et al: Regulation of excision repair cross-complementation group 1 by Snail contributes to cisplatin resistance in head and neck cancer. Clin Cancer Res 16: 4561-4571, 2010.

7. Moody SE, Perez D, Pan TC, et al: The transcriptional repressor Snail promotes mammary tumor recurrence. Cancer Cell 8: 197-209, 2005.

8. Peinado H, Olmeda D and Cano A: Snail, Zeb and bHLH factors in tumour progression: an alliance against the epithelial phenotype? Nat Rev Cancer 7: 415-428, 2007.

9. Debies MT, Gestl SA, Mathers JL, et al: Tumor escape in a Wnt1-dependent mouse breast cancer model is enabled by p19Arf/p53 pathway lesions but not p16 Ink4a loss. J Clin Invest 118: 51-63, 2008.

10. Kudo-Saito C, Shirako H, Takeuchi T, et al: Cancer metastasis is accelerated through immunosuppression during Snail-induced EMT of cancer cells. Cancer Cell 15: 195-206, 2009.

11. Thiery JP, Acloque H, Huang RY, et al: Epithelial-mesenchymal transitions in development and disease. Cell 139: 871-890, 2009.

12. Vincent T, Neve EP, Johnson JR, et al: A SNAIL1-SMAD3/4 transcriptional repressor complex promotes TGF-beta mediated epithelial-mesenchymal transition. Nat Cell Biol 11: 943-950, 2009.

13. Yokoyama K, Kamata N, Hayashi E, et al: Reverse correlation of E-cadherin and snail expression in oral squamous cell carcinoma cells in vitro. Oral Oncol 37: 65-71, 2001.

14. Peinado H, Ballestar E, Esteller M, et al: Snail mediates E-cadherin repression by the recruitment of the Sin3A/histone deacetylase 1 (HDAC1)/HDAC2 complex. Mol Cell Biol 24 306-319, 2004

15. Yang AD, Fan F, Camp ER, et al: Chronic oxaliplatin resistance induces epithelial-to-mesenchymal transition in colorectal cancer cell lines. Clin Cancer Res 12: 4147-4153, 2006.

16. Mani SA, Guo W, Liao MJ, et al: The epithelial-mesenchymal transition generates cells with properties of stem cells. Cell 133: 704-715, 2008.

17. Prince ME, Sivanandan R, Kaczorowski A, et al: Identification of a subpopulation of cells with cancer stem cell properties in head and neck squamous cell carcinoma. Proc Natl Acad Sci USA 104: 973-978, 2007.

18. Sheridan C, Kishimoto H, Fuchs RK, et al: CD44 $/$ CD24- breast cancer cells exhibit enhanced invasive properties: an early step necessary for metastasis. Breast Cancer Res 8: R59, 2006.

19. Visus C, Ito D, Amoscato A, et al: Identification of human aldehyde dehydrogenase 1 family member A1 as a novel CD8 ${ }^{+}$ T-cell-defined tumor antigen in squamous cell carcinoma of the head and neck. Cancer Res 67: 10538-10545, 2007.
20. Mack B and Gires O: CD44s and CD44v6 expression in head and neck epithelia. PLoS One 3: e3360, 2008.

21. Chen YC, Chang CJ, Hsu HS, et al: Inhibition of tumorigenicity and enhancement of radiochemosensitivity in head and neck squamous cell cancer-derived ALDH1-positive cells by knockdown of Bmi-1. Oral Oncol 46: 158-165, 2010.

22. Hayry V, Makinen LK, Atula T, et al: Bmi-1 expression predicts prognosis in squamous cell carcinoma of the tongue. $\mathrm{Br} \mathrm{J}$ Cancer 102: 892-897, 2010.

23. Hayashi K, Motoyama S, Sugiyama T, et al: REG Ialpha is a reliable marker of chemoradiosensitivity in squamous cell esophageal cancer patients. Ann Surg Oncol 15: 1224-1231, 2008.

24. Hayashi Y, Osanai M and Lee GH: Fascin-1 expression correlates with repression of E-cadherin expression in hepatocellular carcinoma cells and augments their invasiveness in combination with matrix metalloproteinases. Cancer Sci 102: 1228-1235, 2011.

25. Al-Hajj M, Wicha MS, Benito-Hernandez A, Morrison SJ and Clarke MF: Prospective identification of tumorigenic breast cancer cells. Proc Natl Acad Sci USA 100: 3983-3988, 2003.

26. Clarke MF, Dick JE, Dirks PB, et al: Cancer stem cells perspectives on current status and future directions: AACR Workshop on cancer stem cells. Cancer Res 66: 9339-9344, 2006.

27. Hermann PC, Huber SL, Herrler T, et al: Distinct populations of cancer stem cells determine tumor growth and metastatic activity in human pancreatic cancer. Cell Stem Cell 1: 313-323, 2007.

28. Li X, Lewis MT, Huang J, et al: Intrinsic resistance of tumorigenic breast cancer cells to chemotherapy. J Natl Cancer Inst 100: 672-679, 2008.

29. Gupta PB, Onder TT, Jiang G, et al: Identification of selective inhibitors of cancer stem cells by high-throughput screening. Cell 138: 645-659, 2009.

30. Charafe-Jauffret E, Ginestier C, Iovino F, et al: Aldehyde dehydrogenase 1-positive cancer stem cells mediate metastasis and poor clinical outcome in inflammatory breast cancer. Clin Cancer Res 16: 45-55, 2010.

31. Locke M, Heywood M, Fawell S, et al: Retention of intrinsic stem cell hierarchies in carcinoma-derived cell lines. Cancer Res 65: 8944-8950, 2005.

32. Okamoto A, Chikamatsu K, Sakakura K, et al: Expansion and characterization of cancer stem-like cells in squamous cell carcinoma of the head and neck. Oral Oncol 45: 633-639, 2009.

33. Bhaijee F, Pepper DJ, Pitman KT, et al: Cancer stem cells in head and neck squamous cell carcinoma: a review of current knowledge and future applications. Head Neck 34: 894-899, 2012.

34. Chen YC, Chen YW, Hsu HS, et al: Aldehyde dehydrogenase 1 is a putative marker for cancer stem cells in head and neck squamous cancer. Biochem Biophys Res Commun 385: 307-313, 2009.

35. Krishnamurthy S, Dong Z, Vodopyanov D, et al: Endothelial cell-initiated signaling promotes the survival and self-renewal of cancer stem cells. Cancer Res 70: 9969-9978, 2010.

36. Schipper JH, Frixen UH, Behrens J, et al: E-cadherin expression in squamous cell carcinomas of head and neck: inverse correlation with tumor dedifferentiation and lymph node metastasis. Cancer Res 51: 6328-6337, 1991.

37. Chung CH, Parker JS, Ely K, et al: Gene expression profiles identify epithelial-to-mesenchymal transition and activation of nuclear factor-kappaB signaling as characteristics of a high-risk head and neck squamous cell carcinoma. Cancer Res 66: 8210-8218, 2006.

38. Bao B, Wang Z, Ali S, et al: Over-expression of FoxM1 leads to epithelial-mesenchymal transition and cancer stem cell phenotype in pancreatic cancer cells. J Cell Biochem 112: 2296-2306, 2011.

39. Chen C, Wei Y, Hummel M, et al: Evidence for epithelial-mesenchymal transition in cancer stem cells of head and neck squamous cell carcinoma. PLoS One 6: e16466, 2011.

40. Fan F, Samuel S, Evans KW, et al: Overexpression of Snail induces epithelial-mesenchymal transition and a cancer stem cell-like phenotype in human colorectal cancer cells. Cancer Med 1: 5-16, 2013.

41. Ota I, Li XY, Hu Y and Weiss SJ: Induction of a MT1-MMP and MT2-MMP-dependent basement membrane transmigration program in cancer cells by Snaill. Proc Natl Acad Sci USA 106: 20318-20323, 2009. 
42. Zhu LF, Hu Y, Yang CC, et al: Snail overexpression induces an epithelial to mesenchymal transition and cancer stem cell-like properties in SCC9 cells. Lab Invest 92: 744-752, 2012.

43. Mendelsohn AH, Lai CK, Shintaku IP, et al: Snail as a novel marker for regional metastasis in head and neck squamous cell carcinoma. Am J Otolaryngol 33: 6-13, 2011.

44. Li L and Neaves WB: Normal stem cells and cancer stem cells: the niche matters. Cancer Res 66: 4553-4557, 2006.

45. Chumsri S, Phatak P, Edelman MJ, et al: Cancer stem cells and individualized therapy. Cancer Genomics Proteomics 4 : $165-174,2007$.

46. Visvader JE and Lindeman GJ: Cancer stem cells in solid tumours: accumulating evidence and unresolved questions. Nat Rev Cancer 8: 755-768, 2008.
47. Boral D and Nie D: Cancer stem cells and niche mircoenvironments. Front Biosci (Elite Ed) 4: 2502-2514, 2012.

48. Calabrese C, Poppleton H, Kocak M, et al: A perivascular niche for brain tumor stem cells. Cancer Cell 11: 69-82, 2007.

49. Gilbertson RJ and Rich JN: Making a tumour's bed: glioblastoma stem cells and the vascular niche. Nat Rev Cancer 7: 733-736, 2007.

50. Yang ZJ and Wechsler-Reya RJ: Hit 'em where they live: targeting the cancer stem cell niche. Cancer Cell 11: 3-5, 2007. 